\title{
Contact angle hysteresis of a drop spreading over metal surfaces
}

\author{
Geniy Kuznetsov ${ }^{1}$, Dmitriy Feoktistov ${ }^{1,2}$ and Evgeniya Orlova ${ }^{1, a}$ \\ ${ }^{1}$ National Research Tomsk Polytechnic University, 30, Lenin Ave, 634050, Tomsk, Russia \\ ${ }^{2}$ Institute of Thermophysics SB RAS, 1, Lavrentiev Ave, 63090, Novosibirsk, Russia
}

\begin{abstract}
The paper presents experimental data on the contact angle hysteresis of the distilled water drop spreading over the surfaces of non-ferrous metals. The measurements of the advancing and receding contact angles were carried out by method of sitting drop on the horizontal surface during increasing and decreasing drop volume with a syringe pump. It was found that the contact line speed has a great influence on the hysteresis of the polished nonelastic substrates. The mechanism of spreading was described using the balance of the forces from the physical point of view.
\end{abstract}

\section{Introduction}

The fundamental characteristic of a drop sitting on a solid surface is the contact angle (CA). According to Young-Laplace equation CA depends only on the surface tensions at the interfaces $\sigma_{S G}, \sigma_{S L}, \sigma_{L G}$. In fact, the angle depends on temperature, shape and arrangements of microasperities, roughness of a surface [1]. The last factor substantially affects the spreading of the droplet with a characteristic size of $1 \mathrm{~mm}$ and less [2,3,4]. Because during spreading over such substrates, a drop pins on microasperities, and it leads to non-uniformly movement of the contact line in all directions. Physical mechanisms of these phenomena have not been studied yet for carrying out the the prognostic assessment. To study these mechanisms, analyses of existing research [5-9] and new results needs to be done.

A contact angle hysteresis is the dependence of the contact angles from the conditions of their formation. Hysteresis can be measured directly by measuring the advancing and receding contact angles (Fig. 1). The advancing contact angle $\theta_{A}$ is the largest angle observed before the contact line begins to move towards the gas phase. The receding contact angle $\theta_{R}$ is characterized by the smallest contact angle observed before the contact line begins to move towards the liquid phase. If the contact line is stationary, the static conact angle $\theta$ can be equal any value between the advancing and receding angles $\theta_{R} \leq \theta \leq \theta_{A}$.

Systematic study of the effect of the surface roughness on the contact angle hysteresis is carried out in [10]. The water drop spreading over surfaces with fluorocarbon and paraffin coatings was under investigation. It was found, that in the initial period of time the dynamic CA increased and the receding CA decreased with increasing the surface roughness. With further increase of the roughness

\footnotetext{
${ }^{\text {a }}$ Corresponding author : lafleur@tpu.ru
} 
the nature of the curve changed: the receding CA increased, the advancing CA raised slowly. At the final stage $\theta_{A}$ is constant with a slight increase of $\theta_{R}$. It leads to small values of the contact angle hysteresis as compared to smooth surfaces.

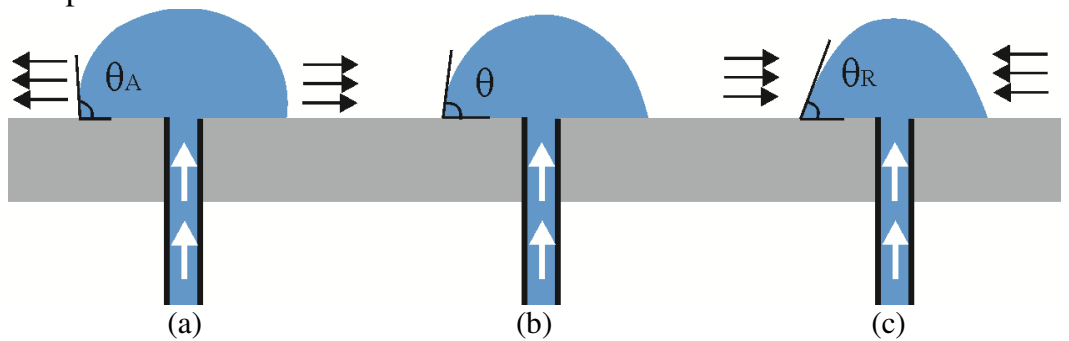

Figure 1. Interpretation of the contact angle hysteresis: (a) advancing contact angle; (b) static contact angle; (c) receding contact angle.

Study of the contact angle hysteresis in different gravity conditions were carried out at spreading a drop over surface with Teflon and polyvinyl-acetate coatings [5]. These surfaces have small and large contact angle hysteresis, respectively. The gravity in the experiment was varied from $0.03 \mathrm{~m} / \mathrm{s}^{2}$ to $1.8 \mathrm{~g}$ ( $\mathrm{g}$ is the terrestrial gravity). The influence of the gravity conditions on drop shape and spreading was observed. The shape of the drop changed from the circular segment under microgravity to flattened profile under hypergravity.

Spreading of a drop on a surface may be realized under the action of external forces, the forced spreading [11, 12], and by placing the droplet on a substrate without application of any forces (gravitational or spontaneous spreading) [13].

Dependences of the advancing contact angle $\left(\theta_{A}\right)$ from the contact line speed $(U)$ were obtained as a result of the forced spreading study $[11,12]$. The nature of the curves is found to change significantly, if one factor changes (for instance, the liquid flow rate). It follows that the contact angle is substantially independent of the contact line speed.

In the second case (gravitational spreading) a drop spontaneously spreads on a surface only by capillary action, the contact angle will decrease, reaching static values in equilibrium state [13]. The speed of the contact line tends to zero.

The measurement of the dynamic CA and the receding CA during forced spreading may be carried out with different contact line speed. For very small speeds (near one $\mu \mathrm{m} / \mathrm{s}$ ) the angles will be close or equal to the static values. However, the nature of the influence of the contact line speed on the hysteresis value has not been established yet. The obtained experimental dependences may subsequently be used as a basis of the drop spreading models verification.

The aim of this study is to determine the effect of the liquid flow rate (the contact line speed) and the material of the substrate to the value of the contact angle hysteresis.

\section{Experimental}

To investigate the dynamics of a drop spreading on substrates, the experimental set-up presented in Figure 2 was used $[14,15]$. The study is based on the use of the shadow optical method for obtaining the drop profiles. The main equipment for the implementation of this method are the source of planeparallel light and high-speed video camera (Fastvideo 500M) with a maximum resolution of $1280 \times 1024$ pixels and with frequency of 10 frames per second, equipped with a macro lens (Sigma AF $105 \mathrm{~mm}$ f/ 2.8 EX DG). 


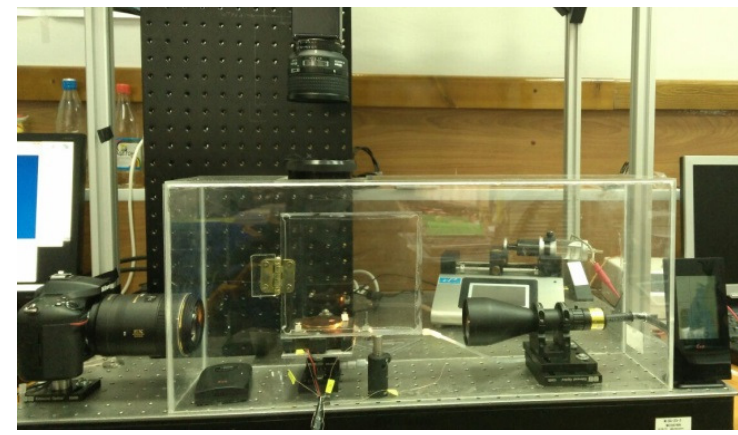

(a)

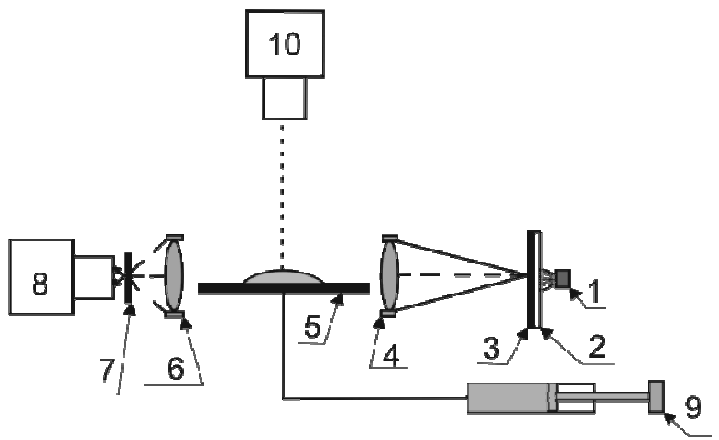

(b)

Figure 2. General view (a) and schematic diagram (b) of an experimental setup: 1 - light source; 2 - ground glass; 3, 7 - transparent shields with an opening; 4 - collimating lens; 5 - substrate; 6 - condensing lens; 8 , 10 high-speed video cameras; 9 - syringe pump.

The second high-speed video camera Fastvideo 500M 10 with lens (Figure 2) was used for visualizing drop formation and controlling drop symmetry. If the drop became asymmetrical due to random influence, the experiment was repeated.

The contact angle hysteresis measurement was carried out by method of sitting drop on the horizontal surface during increasing and decreasing drop volume. To obtain the advancing and the receding contact line, liquid was supplied through the opening in the substrate by an electronic syringe pump. Such nontraditional method of the drop formation (Figure 3) allows implementing the controlled spreading, in particular, changing the liquid flow rate, thereby the contact line speed changes.

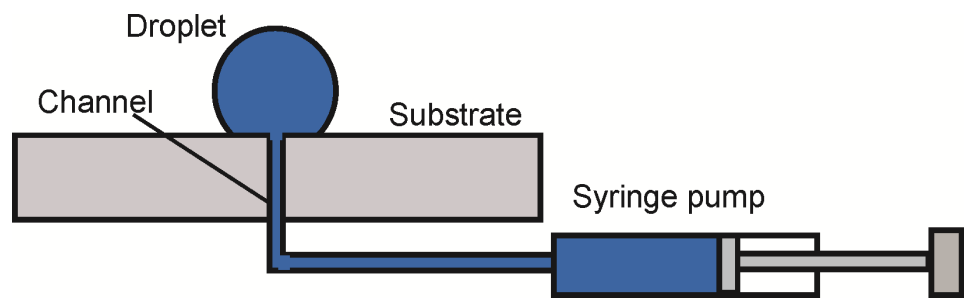

Figure 3. Method of a drop formation by an electronic syringe pump.

A test cell (Figure 4) of the experimental setup consists of the goniometer for lining the surface in two planes and substrate. The disk-shaped substrates $(54 \mathrm{~mm}$ in diameter and $4 \mathrm{~mm}$ thick) made of aluminium and brass with a centered through opening were used in the experiment. The size of the opening was chosen so that it does not affect the wetting of the surface under study. As the wetting liquid nondeaerated distilled water was chosen.

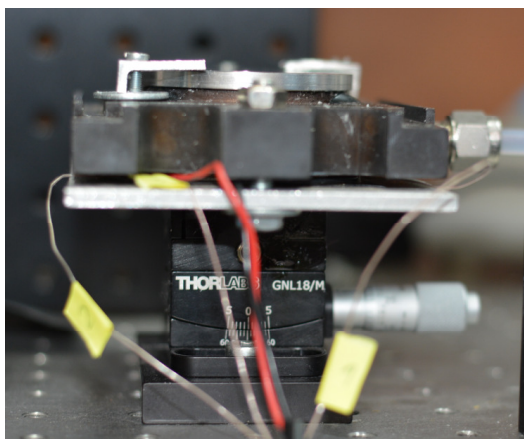

Figure 4. Photo of the test cell. 
The surfaces of the substrates before experimental study were processed on grinding machine with diamond grit. The surface profile and roughness parameter (arithmetic average roughness $\mathrm{Ra}$ ) are obtained on the profilometer "Micro Measure 3D station". Photos of the surfaces are made on scanning electronic microscope Hitachi TM-3000 with multiple zooming (Figures 5, 6).

The flow rate of the liquid supplied through the plastic tube connected with the syringe pump and the opening in the substrate was varied: $0.04 \mathrm{ml} / \mathrm{sec}, 0.05 \mathrm{ml} / \mathrm{sec}, 0.07 \mathrm{ml} / \mathrm{sec}, 0.11 \mathrm{ml} / \mathrm{sec}$.

The main characteristics of spreading drop were obtained after processing shadow images by using software of KRUSS Company. Drop profile was calculated from a shadow photo by using Tangential 1 and Tangential 2 methods.

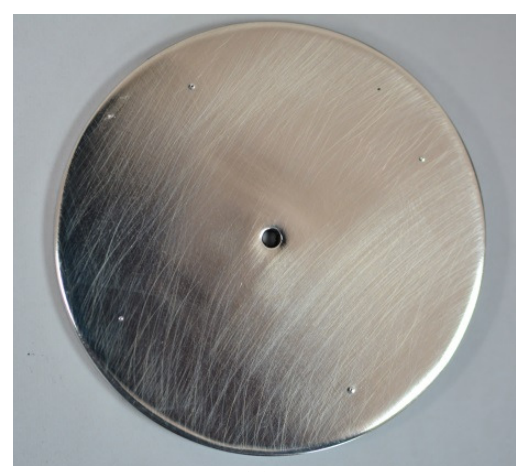

(a)

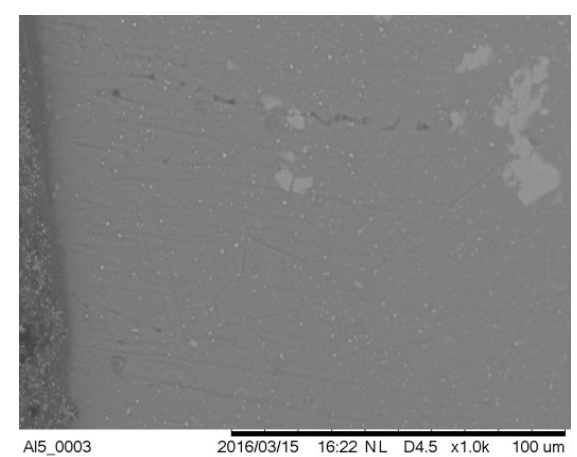

(b)

Figure 5. Aluminium (Ra 0.0287 $\mu \mathrm{m}$ ): (a) - substrate view; (b) - surface microstructure (Magnification: x1000).

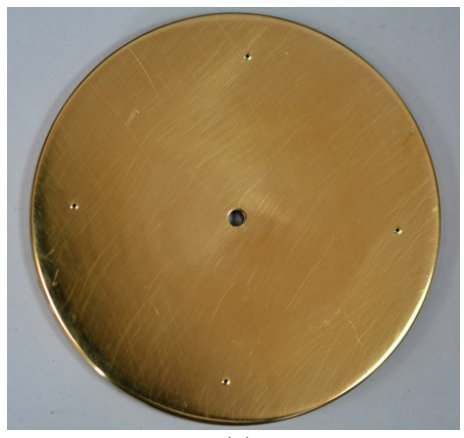

(a)

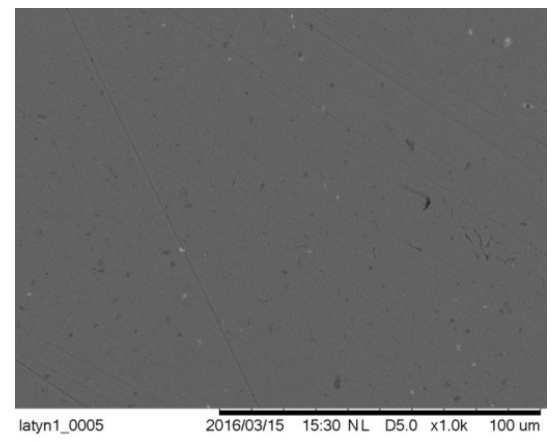

(b)

Figure 6. Brass (Ra $0.033 \mu \mathrm{m}$ ): (a) - substrate view; (b) - surface microstructure (Magnification: x1000).

The main characteristics of spreading drop were obtained after processing the shadow images by using software of KRUSS Company. Drop profile was calculated from a shadow photo by using Tangential 1 and Tangential 2 methods.

Random error was calculated from the results of repeated experiments. To do this, from four to six experiments were carried out at constant values of the studied factors. Thereafter, the mean values of $\theta_{A}, \theta_{R}, U$ and root-mean-square deviations. The fractional error of the contact angle is $2.5 \%$, the error of the contact line speed is $1.9 \%$.

\section{Results and discussion}

As a result of experimental studies the values of the advancing CA and receding CA of the water drop on the surfaces of aluminium and brass substrates. 
Figures 7 and 8 present the dependence of the dynamic contact angle of a drop from the threephase contact line speed on aluminium and brass at different values of the liquid flow rate $(0.04$ $\mathrm{ml} / \mathrm{sec}, 0.05 \mathrm{ml} / \mathrm{sec}, 0.07 \mathrm{ml} / \mathrm{sec}, 0.09 \mathrm{ml} / \mathrm{sec}, 0.11 \mathrm{ml} / \mathrm{sec}$ ). Dependences were plotted according to the values obtained by processing with two different methods: tangential method 1 and tangential method 2. These methods differ in the equations describing the drop profile.

Thus, when the speed $U>0$ contact line moves toward the gas phase, i.e. it is advancing, the advancing contact angle was measured. When the contact line speed $U<0$ the contact line moves toward the liquid phase, i.e. the contact angle is receding. The difference between advancing and receding angles at $U=0$ is the contact angle hysteresis.

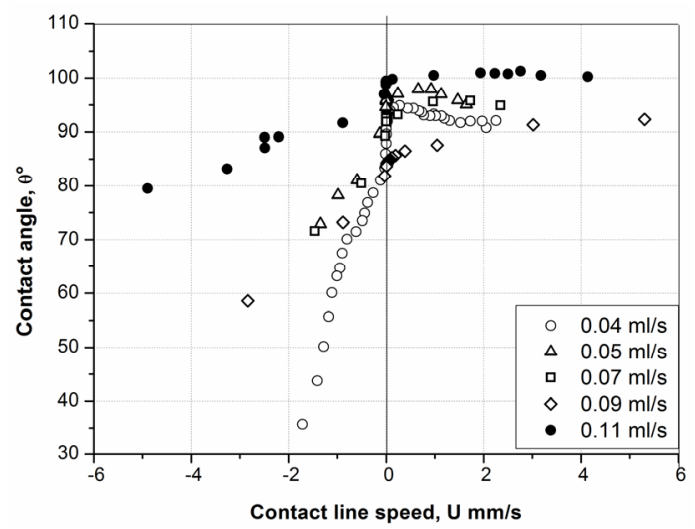

(a)

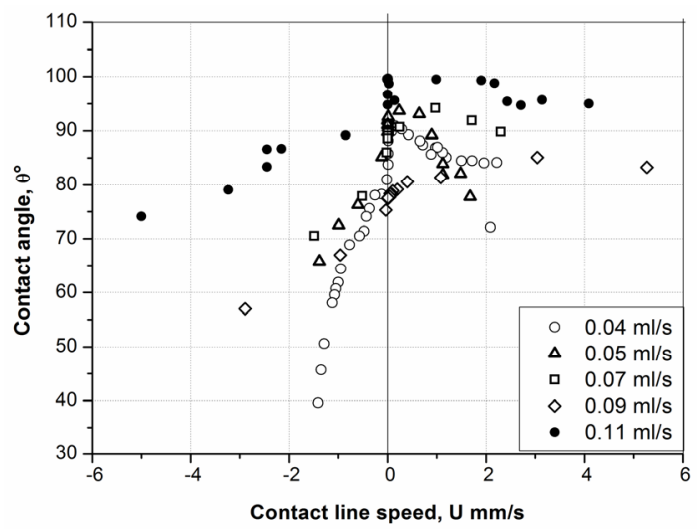

(б)

Figure 7. Dynamic contact angle versus three-phase contact line speed over the substrate made of aluminium with varying liquid flow rate. The results were obtained by two methods of processing: (a) tangential method 1 and (b) tangential method 2.

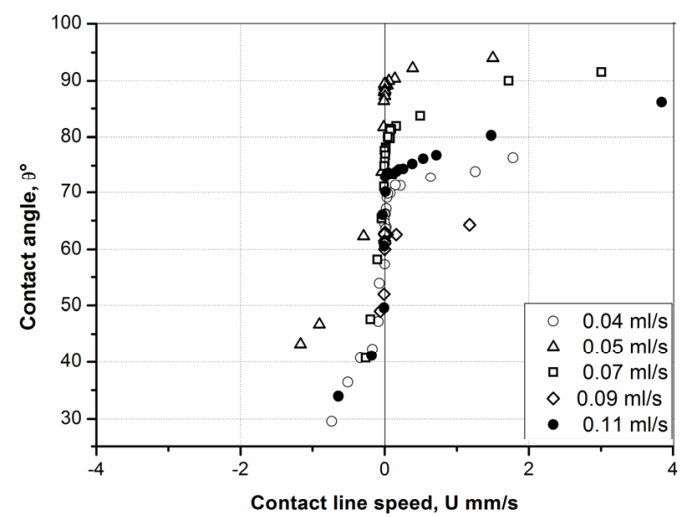

(a)

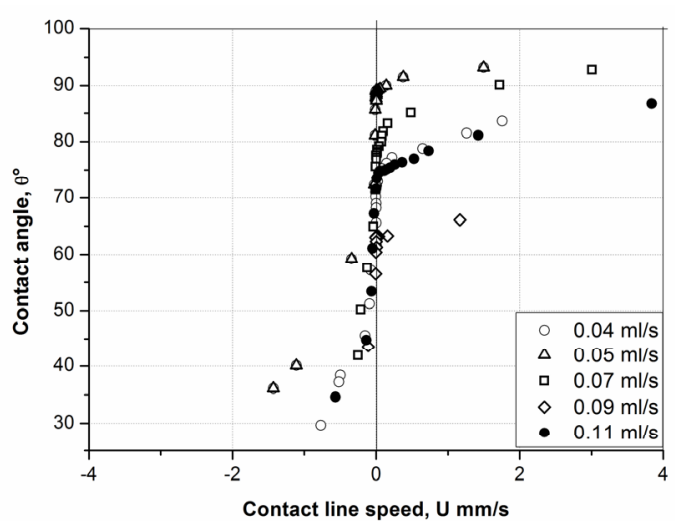

(б)

Figure 8. Dynamic contact angle versus three-phase contact line speed over the substrate made of brass with varying liquid flow rate. The results were obtained by two methods of processing: (a) tangential method 1 and (b) tangential method 2 .

It was found that the contact angle hysteresis changes with varying the liquid flow rate. The obtained values of the hysteresis on aluminium and brass are presented in Tables 1 and 2 .

The contact angle hysteresis also varies depending on the flow rate on the substrate made of brass.

According to the obtained results it was found that the differences in the contact angle hysteresis determined by two methods fall within the limits of the measurement error. 
Table 1. The contact angle hysteresis on aluminium obtained by two methods.

\begin{tabular}{|c|c|c|}
\hline $\begin{array}{c}\text { Liquid flow rate, } \\
\mathrm{ml} / \mathrm{sec}\end{array}$ & \multicolumn{2}{|c|}{ Methods of processing } \\
\cline { 2 - 3 } & Tangential 1 & Tangential 2 \\
\hline 0.04 & $8.2^{\mathbf{o}}$ & $7.4^{\mathbf{o}}$ \\
\hline 0.05 & $1.9^{\mathbf{0}}$ & $2.8^{\mathbf{o}}$ \\
\hline 0.07 & $3.7^{\mathbf{o}}$ & $4^{\mathbf{o}}$ \\
\hline 0.09 & $4^{\mathbf{}}$ & $3.1^{\mathbf{o}}$ \\
\hline 0.11 & $3.7^{\mathbf{}}$ & $4,9^{\circ}$ \\
\hline
\end{tabular}

Table 2. The contact angle hysteresis on brass obtained by two methods.

\begin{tabular}{|c|c|c|}
\hline \multirow{2}{*}{$\begin{array}{c}\text { Liquid flow rate, } \\
\mathrm{ml} / \mathrm{sec}\end{array}$} & \multicolumn{2}{|c|}{ Methods of processing } \\
\cline { 2 - 3 } & Tangential 1 & Tangential 2 \\
\hline 0.04 & $9.9^{\circ}$ & $8.8^{\circ}$ \\
\hline 0.05 & $8^{\circ}$ & $7.7^{\circ}$ \\
\hline 0.07 & $7.2^{\circ}$ & $6.6^{\circ}$ \\
\hline 0.09 & $6.5^{\circ}$ & $5.8^{\circ}$ \\
\hline 0.11 & $6.3^{\circ}$ & $7.5^{\circ}$ \\
\hline
\end{tabular}

It is known [16] that the surface roughness has a great impact on the hysteresis, particularly when microasperities have height not less than $0.5 \mu \mathrm{m}$. The value of hysteresis obtained in the experiment (Tables 1,2 ) is relatively small (less than $10^{\circ}$ ) for substrates with roughness parameter $0.033 \mu \mathrm{m}$ and $0.0287 \mu \mathrm{m}$ for aluminium and brass, respectively. It should be noted that at low contact line speeds the hysteresis is greater than at high speeds (Tables 1,2). Therefore, it may be affirmed that besides the roughness, the contact line speed affects the hysteresis of polished non-elastic solids. As a rule, the value of the speed is caused by the external forces. In this series of experiments, the force depended on the set values of the flow rates on a high-precision syringe pump. Carrying out the analyses of advancing and receding of contact lines, it should be noted that at low contact line speeds the inertial force, which depends on a set flow rate, is smaller in the force balance (the inertia, viscosity, friction, gravity, surface tension), acting on the drop. The inertial forces are small to overcome the energy barriers (occurring due to microasperities and microcavities on the surface of metals). As a result the hysteresis increases. When the flow rate is relatively high, the inertial forces are sufficient to overcome microroughness, the hysteresis in this case will be smaller.

For a visual representation of the physics of advancing and receding contact line on a solid substrate the typical dependences of the dynamic CA from the contact line speed are presented in Figure 9. The advancing contact angle and the contact line speed are found to decrease monotonically during infuse of liquid to a certain value, which is different for brass and aluminium surfaces. Further, after turning off the syringe pump, the contact line does not stop, i.e. the value of the contact angle and the speed continued to decrease. Probably, this is due to the fact that the drop has continued to spread under the influence of the residual pulse due to the hydrodynamic flows in the drop volume. Spreading in this period may be called inertial or spontaneous, which lasts until the establishment of equilibrium state. Then, a syringe pump is switched on and the liquid is withdrawn for the receding contact line formation, accompanied by a decrease in the receding contact angle and the contact line speed. 


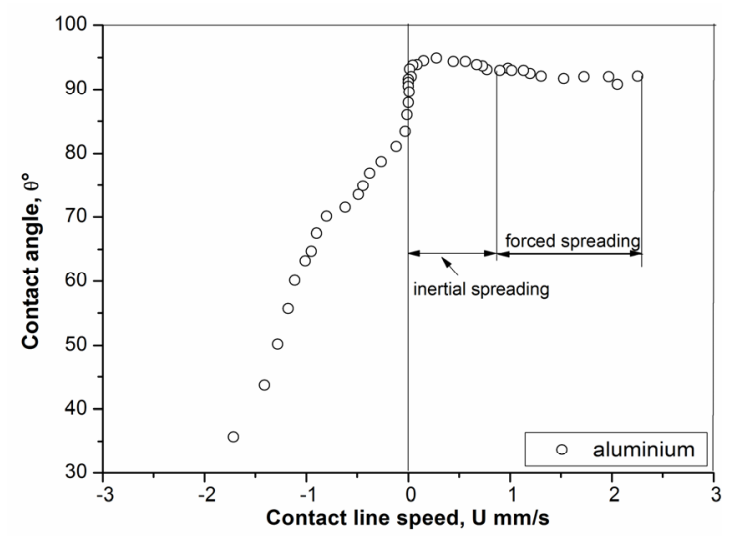

(a)

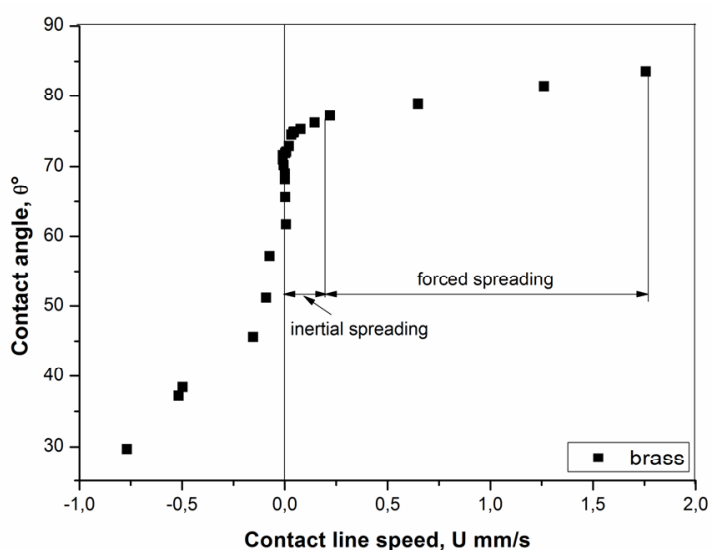

(b)

Figure 9. Typical experimental results for the dependence of the dynamic contact angle on the contact line speed for the liquid flow rate of $0.04 \mathrm{ml} / \mathrm{s}$ on the substrates made of: (a) aluminium and (b) brass.

\section{Conclusion}

According to results of the experimental study it was found the effect of the liquid flow rate on the contact angle hysteresis of polished metal substrates. In addition to the surface roughness, the contact line speed significantly affects the hysteresis. The influence of the inertia forces on the hysteresis of non-elastic solids was explained. The mechanism of spreading was described from the physical point of view according to obtained dependences of the dynamic CA and receding CA from the contact line speed.

It was found that the differences in the contact angle hysteresis determined by two methods (tangential 1 method and tangential 2 method) fall within the limits of the measurement error.

The reported study was supported by the grant of the President of the Russian Federation for the government support of young Russian scientists (MK-6810.2016.8).

\section{References}

1. P.G. de Gennes, Rev. Mod. Phys. 57, 827 (1985)

2. D. Sivakumar, K. Katagiri, T. Sato, H. Nishiyama, Phys. Fluids 17, 100608 (2005)

3. H. Song, Y. Lee, S. Jin, H.-Y. Kim, J.Y. Yoo, Microelectron Eng 88, 3249 (2011)

4. V.S. Ajaev, E.Ya. Gatapova, O.A. Kabov, Adv. Colloid Interface Sci. 228, 92 (2016)

5. Zaitsev D.V., Kabov O.A., Microgravity science and technology, 19, 174 (2007).

6. D. Bonn, J. Eggers, J. Indekeu, J. Meunier, E. Rolley, Rev. Mod. Phys. 81, 739 (2009)

7. C.L. Moraila-Martinez, F.J. Montes Ruiz-Cabello, M.A. Cabrerizo-Vilchez, M.A. RodriguezValverde, Colloids Surf., A 404, 63 (2012)

8. G. McHale, N.J. Shirtcliffe, M.I. Newton, Langmuir 20, 10146 (2004)

9. J.W. Krumpfer, T.J. McCarthy, Faraday Discuss. 146, 103 (2010)

10. R.H. Dettre, R.R. Johnson jr. Contact Angle, Wettability and Adhesion (American Chemical Society, Washington, DC, 1963)

11. T.D. Blake, A. Clarke, K.J. Ruschak, AIChE J. 40, 229 (1994)

12. T.D. Blake, M. Bracke, Y.D. Shikhmurzaev. Phys. Fluids 11, 1995 (1999)

13. M.J. de Ruijter, J. De Coninck, T.D. Blake, A. Clarke, A. Rankin, Langmuir 13, 7293 (1997)

14. E.G. Orlova, G. V. Kuznetsov, D. V. Feoktistov, EPJ Web of Conferences 82, 01053 (2015)

15. D. Feoktistov, E. Orlova, A. Islamova, EPJ Web of Conferences 110, 01018 (2016)

16. B.D Summ, Yu.V. Goryunov, The Physochemical Principles of Wetting and Spreading [in Russian] (Khimiya, Moscow, 1976) 